\title{
Real-time image processing via four-wave mixing in a photorefractive medium
}

\author{
Jeffrey O. White and Amnon Yariv \\ California Institute of Technology, Pasadena, California 91125
}

(Received 21 January 1980; accepted for publication 14 April 1980)

\begin{abstract}
We report the application of four-wave mixing to real-time image processing. We constructed a nonlinear optical processor using the photorefractive medium $\mathrm{Bi}_{12} \mathrm{SiO}_{20}$ and demonstrated that it is capable of convolving and correlating objects with spatial information.
\end{abstract}

PACS numbers: 42.65. $-\mathrm{k}, 42.40 .-\mathrm{i}, 42.30 . \mathrm{Kq}, 42.30 . \mathrm{Sy}$

In recent years, coherent and incoherent image processing has been used for pattern recognition, guidance systems, deblurring, and synthetic aperturing. Present methods used to generate convolution and correlation functions of objects with spatial information include digital processing, Van der Lugt-type holograms, ${ }^{1,2}$ and three-wave mixing. ${ }^{3}$ The latter scheme requires attention to phase matching, which also limits the spatial bandwidth of the information. The use of multiple wavelengths introduces spatial scaling, which may be objectionable. A new method utilizing four-wave mixing was proposed by Pepper, Au Yeung, Fekete, and Yariv. ${ }^{4}$ This method works in real time and was shown to be free of the phase matching problem, in the Fresnel approximation, and to require only a single frequency. We report the demonstration of such an optical processor and show some sample results.

Spatial convolution and correlation of monochromatic fields can be obtained via four-wave mixing in the common focal plane of a two-lens system such as that shown in Fig. 1. Fields 1 and 4 propagate essentially in the $z$ direction, while 2 propagates in the $-z$ direction. All three fields, which are of the same frequency, may contain arbitrary spatial modulation which is different for each wave. Using the notation of Ref. 4 , the input amplitudes $u_{1}(x, y), u_{2}(x, y)$, and $u_{4}(x, y)$ in the outer focal planes are Fourier transformed by propagating to the common focal plane. The transformed fields $\tilde{u}_{1}, \tilde{u}_{2}$, and $\tilde{u}_{4}$ are incident upon a medium of thickness $l$ possessing a third-order nonlinear optical susceptibility $\chi_{\mathrm{NL}}^{(3)}$. The thickness must satisfy

$$
l<2 f^{2} \lambda / r_{\max }^{2},
$$

where $r_{\max }$ is the spatial extent of the largest $u_{i}$, so that the mixing only occurs in the region where the fields are accurate Fourier transforms of their respective input fields. ${ }^{4}$

The nonlinear polarization $P_{\mathrm{NL}}=\chi_{\mathrm{NL}}^{(3)} \tilde{u}_{1} \tilde{u}_{2} \tilde{u}_{4}^{*}$ which is formed in the medium radiates an output field which propagates essentially backward relative to the direction of $u_{4}$. It can be shown that this field, when evaluated at the plane located a distance $f$ in front of lens $L$, is of the form

$u_{3}\left(x_{0}, y_{0}\right)=\psi u_{1}(-x,-y) * u_{2}(-x,-y) \star u_{4}(-x,-y)$,

where

$$
\psi=-i(2 \pi \omega / c)\left(l / \lambda^{2} f^{2}\right) \exp (2 i k n \Delta) \exp (4 i k f) \chi_{\mathrm{NI}}^{(3)}
$$

and $\Delta$ is the thickness of the lens. The symbols * and * denote convolution and correlation, respectively. ${ }^{2}$

The nonlinear medium used in our experiment was $\mathrm{Bi}_{12} \mathrm{SiO}_{20}$ (BSO), a photorefractive material. It has been used in Pockels readout optical modulators, ${ }^{5}$ holographic storage $^{6}{ }^{6}$ and phase conjugation. ${ }^{7}$ The analogy between fourwave mixing and real-time holography ${ }^{8}$ suggested the use of BSO for our experiment. The susceptibility $\chi_{\mathrm{NL}}^{(3)}$ in the nonlinear theory can be related to the amplitude $n_{1}$ of the index variation which characterizes a phase hologram by comparing the coupled mode equations in the formulations of Yariv and Pepper, ${ }^{9}$ and Kogelnik ${ }^{10}$ :

$$
n_{1}=-\left(4 \pi / n_{0}\right) \chi_{\mathrm{NL}}^{(3)} A_{1} A_{4}^{*} \text {. }
$$

The formation of phase holograms in photorefractive materials has been studied extensively. "The situation usually analyzed is one where two plane waves, with angular separation $2 \theta$, interfere inside the medium to produce a sinusoidal intensity pattern with grating vector $k=4 \pi(\sin \theta) / \lambda$. The excitation of electrons from traps in the energy gap to the conduction band is assumed to be proportional to the intensity. Drift (due to an external dc field) and diffusion of the free carriers relative to the positively ionized traps sets up a periodic space-charge field $E_{\mathrm{SC}}$. The amplitude $E_{1}$ of the fundamental component has been shown experimentally ${ }^{6,7}$ and theoretically ${ }^{12}$ to be proportional to an external field $E_{0}$ applied perpendicular to the intensity fringes, and to the fringe modulation $m=\left(I_{1} I_{4}\right)^{1 / 2} /\left(I_{1}+I_{4}\right)$.

The electro-optic effect then modulates the index of refraction with a fundamental period equal to that of the intensity pattern. The phase shift of the index modulation relative to the intensity modulation depends on the writing time and on the relative contributions of drift and diffusion. ${ }^{13}$ The amplitude of the fundamental component of the index modulation will be

$$
n_{1}=\frac{1}{2} n_{0}^{3} r E_{1} \propto \frac{1}{2} n_{0}^{3} r m E_{0} \propto n_{0}^{3} r E_{0}\left(I_{1} I_{4}\right)^{1 / 2} / 2\left(I_{1}+I_{4}\right) .
$$

In our experiment, the three beams incident on the crystal are coplanar and linearly polarized perpendicular to that plane. They form three interference patterns, but the optical activity rotates polarizations so as to almost completely reduce the interference involving $u_{2}$. Also, the application of a transverse (perpendicular to $z$ ) $E_{0}$ enhances only the intefer- 


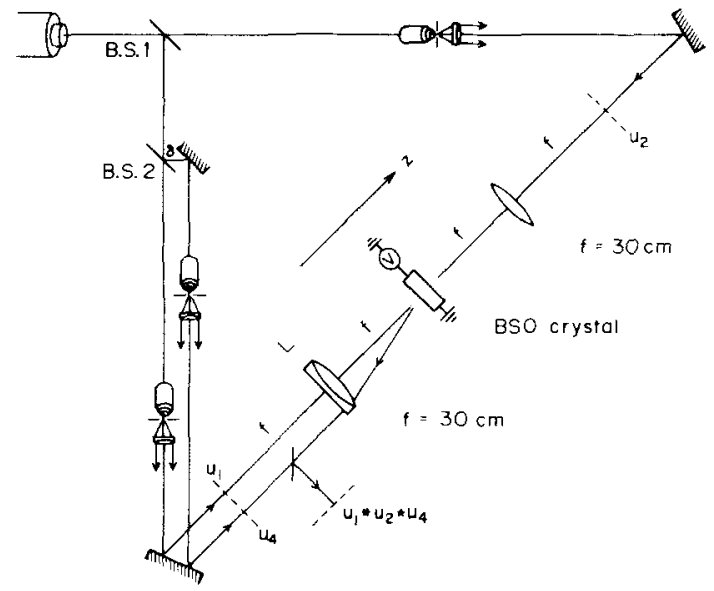

FIG. 1. Experimental apparatus for performing convolution and correlation. Input and output planes shown by dashed lines.

ence pattern between $u_{1}$ and $u_{4}$. The reconstruction beam is then $u_{2}$. If the intensity of the backward beam $u_{3}$ were high, we would also have to consider the interference between $u_{2}$ and $u_{3}$.

Using Kogelnik's formula for diffraction efficiency and including reflection losses at the crystal surface, the output intensity is given by

$$
\begin{aligned}
I_{3} & =\eta I_{2}(1-R)^{2}=\exp (-\alpha l) \sin ^{2}\left(\frac{\pi l n_{1}}{\lambda \cos \theta_{0}}\right) I_{2}(1-R)^{2} \\
& \propto \exp (-\alpha l)\left(\frac{\pi l}{\lambda}\right)^{2}\left(\frac{n_{0}^{3} r E_{0}}{2}\right)^{2} \frac{I_{1} I_{4} I_{2}}{\left(I_{1}+I_{2}+I_{4}\right)^{2}}(1-R)^{2}
\end{aligned}
$$

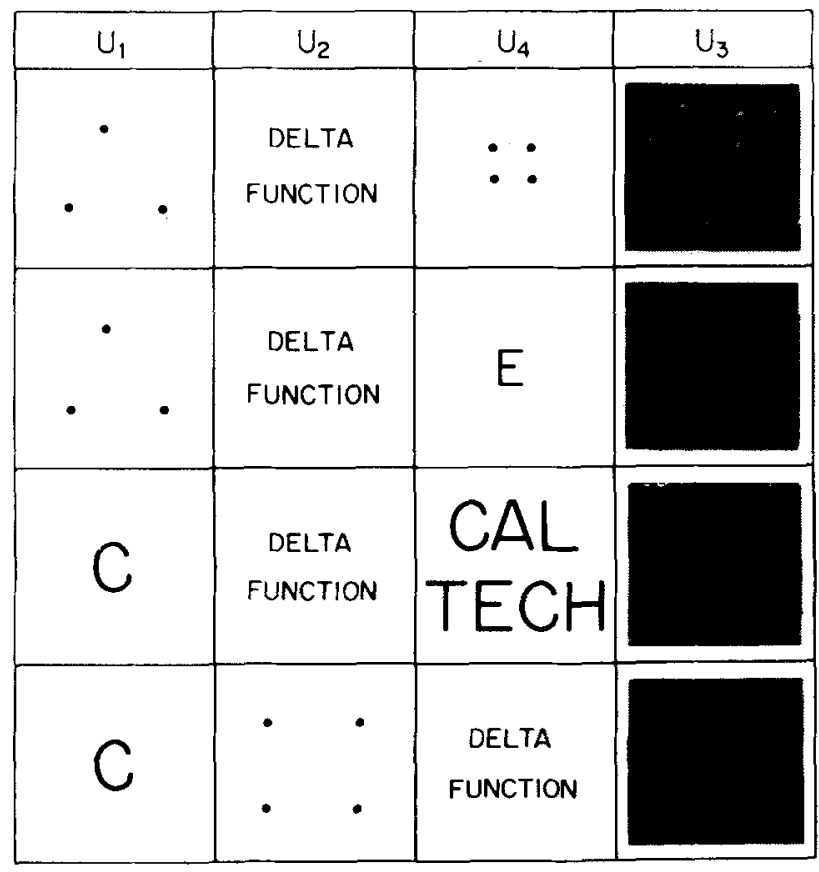

FIG. 2. First three columns are the input objects $u_{1}, u_{2}$ and $u_{4}$. The last column is photographs of the output $u_{\gamma}$.
In our case the Bragg angle $\theta_{0} \simeq 0$ for small enough objects. For a given power output of the laser, the power diffracted by the crystal is seen to.be maximized when $I_{1}=I_{2}=I_{4}$.

The experimental arrangement is shown in Fig. 1. The three beams travel nearly equal path lengths before impinging on the BSO crystal. The coherence length of the laser must be greater than the optical path difference, in this case $\delta$. The cw argon laser has a power output of $1.6 \mathrm{~W}$ at $5145 \AA$ initially polarized perpendicular to the plane of the figure.

The BSO crystal is $10 \times 10 \times 3 \mathrm{~mm}$. The applied voltages ranged from 5 to $7 \mathrm{kV}$. Photographs of the output were taken by placing ASA 400 black and white film directly in the output plane. Exposure times were 1/500 sec. The objects we used included photographic negatives, chrome photomasks, perforated $\mathrm{Al}$ foil, and lenses.

Objects of different sizes were accomodated by expanding each beam to the appropriate waist. As seen in Eq. (2), the output of the processor is optimal when the variable beamsplitters, B.S. 1 and B.S. 2, are adjusted to match the intensities $I_{1}, I_{2}$ and $I_{4}$ where they overlap in the transform plane.

Two-image processing is performed by simulating a $\delta$ function for the third input. The simulation is accomplished by placing a third lens outside the two-lens system so that it focuses at the third input plane and gives a collimated beam at the transform plane. For objects with real amplitude transmittances $u_{i}$, the correlation and convolution operations differ only in the sign of the arguments ${ }^{2}$ so that correlation can be done by convolving the inverted object and vice versa.

Typical experimental results are shown in Fig. 2. The first three columns show the input fields $u_{1}, u_{2}$, and $u_{4}$. The fourth column shows the photograph obtained at the output plane. Rows (a)-(c) illustrate correlation and row (d) illustrates convolution. The input information in (a) and (b) is

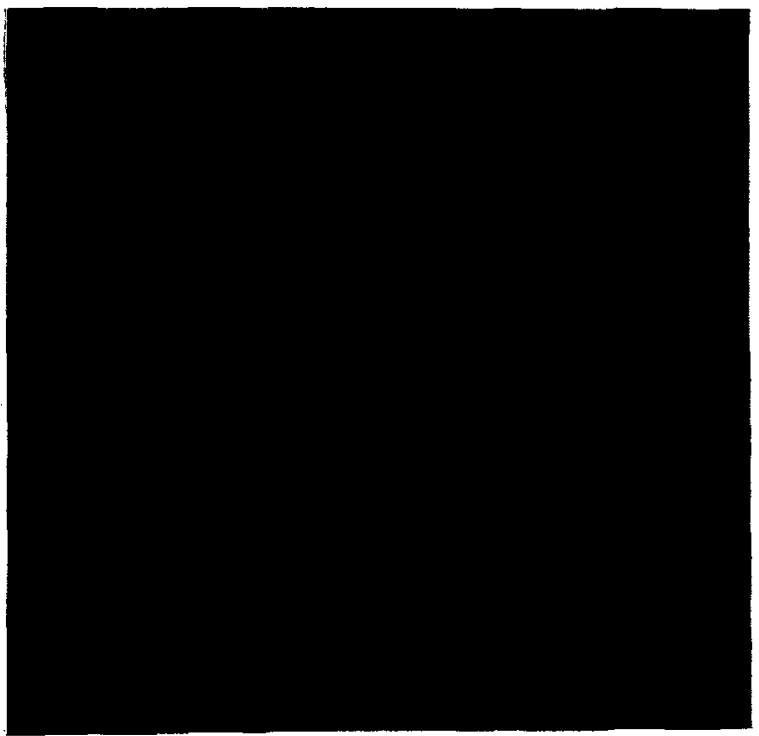

FIG. 3. Output when the inputs are a resolution chart and two $\delta$ functions 
TABLE I. Measurements of output wavefront radius of curvature when lenses are used as inputs.

\begin{tabular}{|c|c|c|c|}
\hline \multicolumn{2}{|c|}{$f(\mathrm{~cm})$} & \multirow[b]{2}{*}{$\begin{array}{l}\text { Output } \\
\text { wavefront }\end{array}$} & \multirow[b]{2}{*}{$\begin{array}{l}\text { Radius of } \\
\text { curvature }(\mathrm{cm})\end{array}$} \\
\hline $\begin{array}{l}\text { Lens at input } \\
\text { plane } 1\end{array}$ & $\begin{array}{l}\text { Lens at input } \\
\text { plane } 4\end{array}$ & & \\
\hline 33.3 & 50.0 & diverging & $\ldots$ \\
\hline 33.3 & -9.5 & converging & 41.4 \\
\hline 33.3 & -50.5 & converging & 81.0 \\
\hline 33.3 & 20.0 & converging & 13.2 \\
\hline
\end{tabular}

cut in $\mathrm{Al}$ foil. The inputs in (c) and (d) are etched in chrome photomasks. The triangular and square clusters are 75-100 $\mu \mathrm{m}$-diam pinholes spaced $0.7-1 \mathrm{~mm}$ apart. The letters are $1.2 \mathrm{~mm}$ high. The output in row (c) clearly demonstrates pattern recognition. The output in row (d) is inverted in agreement with Eq. (1). Figure 3 shows the output when a resolution chart was inserted in $u_{2}$ and $\delta$ functions were used for $u_{1}$ and $u_{4}$. The signal-to-noise ratio was increased by using a Polaroid filter in the output plane to eliminate scattered light. A half wave plate can be inserted in $\boldsymbol{u}_{2}$ in such a way as to bring the polarization of the slightly elliptical $u_{3}$ beam perpendicular to that of the scattered light.

The operation of the processor was also tested with purely phase objects. For inputs we used thin lenses having amplitude transmittances $t \sim \exp \left[-j k\left(x^{2}+y^{2}\right) / 2 f\right]$. If two such lenses are correlated, the $x$ and $y$ dependence of the field in the output plane should be equivalent to that due to a lens having focal length equal to the difference in the input focal lengths, illuminated by a plane wave:

$$
u_{1} \star u_{4} \sim \frac{-j \pi}{k\left(1 / f_{1}-1 / f_{2}\right)} \exp \left(\frac{-j k}{2} \frac{x^{2}+y^{2}}{f_{1}-f_{4}}\right) .
$$

We determined the general curvature of the output wave by simply looking for a focus at a distance $f_{1}-f_{4}$ behind the output plane. Table I summarizes the measured results, which agree well with the prediction.

The versatility of this processor allows all three inputs to be time varying, but we measured the hologram writing time to be $30 \mathrm{msec}$ (with an applied field of $5 \mathrm{kV} / \mathrm{cm}$ ) so that rapidly changing input fields will cause the output to decrease. Another practical problem is that the range of input light intensities is limited by the saturation effect in BSO. The combination of sensitivity to both the amplitude and the phase of the input fields indicates that the correlation processor might be useful for applications such as screening tissue samples for cancer cells.

The authors would like to thank our colleague $\mathrm{Y} . \mathrm{Ku}$ bota for many helpful discussions and technical assistance. We also thank the Army Research Office in Durham, N. C. for their financial support.

\footnotetext{
'A. B. Van der Lugt, IEEE Trans. Inf. Theory IT-10, 2 (1964).

${ }^{2}$ J. W. Goodman, Introduction to Fourier Optics (McGraw-Hill, New York, 1968 ).

${ }^{3}$ R. A. Eremeeva, V. A. Kudryashov, I. N. Matrieev, T. G. Usacheva, and A. I. Chekemenev, Sov. J. Quantum Electron. 7, 90 (1977).

${ }^{4}$ D. M. Pepper, J. Au Yeung, D. Fekete, and A. Yariv, Opt. Lett. 3, 7 (1978).

5S. L. Hou and D. S. Oliver, Appl. Phys. Lett. 18, 325 (1971).

${ }^{6} J$. P. Huignard and F. Micheron, Appl. Phys. Lett. 29, 591 (1976).

JJ. P. Huignard, J. P. Herriau, P. Auborg, and E. Spitz, Opt. Lett. 4, 21 (1979).

${ }^{x}$ A. Yariv, J. Opt. Soc. Am. 66, 301 (1976).

${ }^{9}$ A. Yariv and D. M. Pepper, Opt. Lett. 1, 16 (1977).

${ }^{116} \mathrm{H}$. Kogelnik, Bell Syst. Tech. J. 48, 2909 (1969)

"A. M. Glass, Opt. Eng. 17, 470 (1978).

'2D. M. Kim, R. R. Shah, T. A. Rabson, and F. K. Tittel, Appl. Phys. Lett. 28, 338 (1976).

${ }^{13}$ N. V. Kukhtarev, V. B. Markov, S. G. Odulov, M. S. Soskin, and V. L.
} Vinetskii, Ferroelectrics 22, 949 (1979).

\title{
Liquid-phase-epitaxy homostructure $\mathrm{Pb}_{0.85} \mathrm{Sn}_{0.15}$ Te diode laser with controlled carrier concentration
}

\author{
M. Oron and A. Zussman \\ Solid State Physics Department, Soreq Nuclear Research Centre, Yaune, Israel
}

(Received 11 March 1980; accepted for publication 1 April 1980)

Homostructure $\mathrm{Pb}_{0.85} \mathrm{Sn}_{0.15}$ Te diode lasers were fabricated from a liquid-phase-epitaxy grown $n^{+}-p-p^{+}$layer structure. Pulsed threshold currents as low as $40 \mathrm{~A} / \mathrm{cm}^{2}$ at $17^{\circ} \mathrm{K}$ and $1.5 \mathrm{kA} / \mathrm{cm}^{2}$ at $77^{\circ} \mathrm{K}$ were measured. High external and internal quantum efficiencies of 17 and $34 \%$, respectively, were found at $40^{\circ} \mathrm{K}$. No degradation of the threshold current was detected after fifteen thermal cycles.

PACS numbers: $42.55 . \mathrm{Rz}$

The introduction of a double heterostructure (DH) configuration improved the performance of $\mathrm{PbSrTe}$ diode lasers, compared with that of homostructure lasers, at high temperatures. ${ }^{1-3}$ The reduction in the threshold current den- sity in DH lasers at high temperatures made possible $\mathrm{cw}$ operation above $77^{\circ} \mathrm{K} .{ }^{1.4}$ However, at low temperatures DH lasers have threshold currents of one order of magnitude higher than the homostructure lasers. ${ }^{1-3} \mathrm{DH}$ lasers also suf- 